\title{
On the Strominger System and Holomorphic Deformations
}

\author{
Stefan Ivanov ${ }^{1,2}$. Luis $\operatorname{Ugarte}^{3}$
}

Received: 21 December 2017

(C) Mathematica Josephina, Inc. 2018

\begin{abstract}
We prove that the property of existence of solution to the Strominger system in dimension six is neither open nor closed under holomorphic deformations of the complex structure. These results are obtained both in the case of positive slope parameter as well as in the case of negative slope parameter in the anomaly cancellation equation.
\end{abstract}

Keywords Complex structure · Holomorhic deformation - Balanced Hermitian metric $\cdot$ Strominger system

Mathematics Subject Classification Primary 32G05; Secondary 53C55 - 53C07 . $81 \mathrm{~T} 30$

\section{Introduction}

Let $X$ be a compact Kähler manifold with holomorphically trivial canonical bundle. By Yau's solution to the Calabi conjecture [43], $X$ admits a Ricci-flat Kähler metric. However, there are many compact complex manifolds $X$ with holomorphically trivial

\footnotetext{
$凶$ Luis Ugarte

ugarte@unizar.es

Stefan Ivanov

ivanovsp@fmi.uni-sofia.bg

1 Faculty of Mathematics and Informatics, University of Sofia “St. Kl. Ohridski”, Blvd. James Bourchier 5, 1164 Sofia, Bulgaria

2 Institute of Mathematics and Informatics, Bulgarian Academy of Sciences, Sofia, Bulgaria

3 Departamento de Matemáticas - I.U.M.A., Universidad de Zaragoza, Campus Plaza San Francisco, 50009 Zaragoza, Spain
} 
canonical bundle not admitting any Kähler metric, and it arises the question of existence of canonical Hermitian metrics in this setting. Candidates for dealing with this problem are the solutions to the Strominger system. In [39], Strominger investigated the heterotic superstring background with non-zero torsion, which led to a complicated system of partial differential equations. Roughly speaking, the system requires $X$ to be a compact complex conformally balanced manifold with holomorphically trivial canonical bundle, equipped with an instanton $A$ satisfying the anomaly cancellation equation

$$
d T=\frac{\alpha^{\prime}}{4}\left(\operatorname{tr} \Omega \wedge \Omega-\operatorname{tr} \Omega^{A} \wedge \Omega^{A}\right),
$$

for a non-zero constant $\alpha^{\prime}$ which is the slope parameter in string theory. Here, $T$ denotes the 3 -form defined by the torsion of the Strominger-Bismut connection of the Hermitian metric, $\Omega$ is the curvature form of some metric connection $\nabla$ and $\Omega^{A}$ is the curvature form of the instanton $A$, i.e., $\Omega^{A}$ is the curvature form of a connection on a holomorphic hermitian vector bundle $(E, h)$ over $X$ which satisfies the HermitianYang-Mills equation.

Any Kähler Calabi-Yau manifold solves the Strominger system taking e.g., $\Omega^{A}=$ $\Omega$, and in the last years many efforts have been made in constructing solutions on non-Kähler manifolds, more specifically in complex dimension 3. In [30], Li and Yau obtained the first non-Kähler solutions to the Strominger system on a Kähler Calabi-Yau manifold, which was further extended in [3]. Based on a construction in [27], Fu and Yau proved the existence of solutions to the Strominger system on nonKähler Calabi-Yau manifolds given as a $\mathbb{T}^{2}$-bundle over a $K 3$ surface [23,24], see [34] for solutions in all dimensions. Recently, Phong, Picard, and Zhang investigating the anomaly flow in [35] recapture the Fu-Yau results. Explicit smooth solutions to the Strominger system on compact non-Kähler Calabi-Yau 3-folds with infinitely many topological types and sets of Hodge numbers are constructed very recently in [19] relying on the generalized Calabi-Gray construction outlined in [17]. Explicit solutions on nilmanifolds have been constructed in [12,20,21,42], and more recently invariant solutions were also found on some solvmanifolds and on compact quotient of SL(2, $\mathbb{C})$ in $[18,33]$, see also [4]. On the other hand, some recent developments in the infinitesimal moduli problem of the Strominger system were presented in $[15,16]$ and the relation of the Strominger system with generalized geometry is obtained for instance in [2,26], see the notes [25] for more results on the subject.

Solutions of the Strominger system under deformations of the SU(3) structure, i.e., holomorphic deformations of the complex structure together with a deformation of the conformally balanced condition, are investigated by de la Ossa and Svanes in [14], see also [7] where the authors consider deformations of the complex structure only. It is shown in [14] that under deformations of the complex structure alone the conformally balanced condition gives rise to a complicated PDE which seems to be a difficult equation to satisfy. In this note, we confirm in some sense this difficulty.

We say that a compact complex manifold $X$ has the Strominger property if $X$ admits a solution to the Strominger system (see Definition 2.1). Any Kähler Calabi-Yau manifold has the Strominger property, and any $X$ satisfying the Strominger property is in particular a balanced Hermitian manifold. By the well-known Bogomolov- 
Tian-Todorov theorem (announced in [11] and proved independently in [40,41]), the deformation space of any Kähler Calabi-Yau manifold is unobstructed. In contrast to this case, where any sufficiently small deformation of a Kähler Calabi-Yau manifold is again Kähler Calabi-Yau, it was proved in [1] by Alessandrini and Bassanelli, relying on Michelsohn's investigations in [32], that the balanced property is not stable under small deformations of the complex structure. Since the compact complex manifolds $X$ satisfying the Strominger property constitute an important class between the class of Kähler Calabi-Yau manifolds and the class of balanced manifolds, it arises the natural question of whether that class is stable or not under holomorphic deformations of the complex structure.

Our first goal in this paper is to prove that the Strominger property is not stable. We will distinguish two cases depending on the sign of the slope parameter $\alpha^{\prime}$ in (1.1). Furthermore, we will also prove that the Strominger property is not closed, i.e., the central limit of a holomorphic family of solutions of the Strominger system may not admit any solution.

The paper is structured as follows. In Sect. 2, we recall some terminology and results on the Strominger system. In Sect. 3, we construct a holomorphic family $\left(X_{\mathbf{t}}\right)_{\mathbf{t} \in \Delta}$ such that $X_{0}$ admits a solution to the Strominger system with positive $\alpha^{\prime}$, but there does not exist any balanced metric on $X_{\mathbf{t}}$ for arbitrary small values of $\mathbf{t} \neq 0$ (see Proposition 3.1 and Theorem 3.2). Hence, the Strominger property with positive slope parameter $\alpha^{\prime}$ is not stable. For the construction of such a family, we first analyze the space of invariant complex structures on the manifold underlying the Iwasawa manifold, which leads us to consider a compact complex manifold $X_{0}$ satisfying certain specific condition on its Bott-Chern cohomology group of bidegree $(2,2)$.

In Sect. 4, we prove that the Strominger property with positive slope parameter $\alpha^{\prime}$ is not closed under holomorphic deformations. Indeed, there is a holomorphic family of compact complex manifolds $\left(X_{\mathbf{t}}\right)_{\mathbf{t} \in \Delta}$ such that $X_{\mathbf{t}}$ has a solution to the Strominger system with $\alpha^{\prime}>0$ for any $\mathbf{t} \in \Delta-\{0\}$, but there does not exist any such solution on its central limit $X_{0}$ (see Theorem 4.1). To prove this result, we further study the family $\left(X_{\mathbf{t}}\right)_{\mathbf{t} \in \Delta}$ constructed in [13] to answer a question in [36] about the closedness of the balanced property. We prove that for any $\mathbf{t} \neq 0$, the manifold $X_{\mathbf{t}}$ has a solution to the Strominger system with $\alpha^{\prime}>0$. Hence, the non-closedness result comes from the fact that the central limit $X_{0}$ does not admit any balanced metric.

Section 5 is devoted to the negative slope parameter case, and we show that again the Strominger property with $\alpha^{\prime}<0$ is neither open nor closed under holomorphic deformations. It should be remarked that the results obtained in Sects. 3 and 4 for $\alpha^{\prime}>0$ cannot be applied directly to the $\alpha^{\prime}<0$ case. However, we make use of a non-flat instanton $A$ constructed in [12] to reverse the sign of the slope parameter in the solutions found in Sects. 3 and 4.

\section{The Strominger System}

The Strominger system [28,39] is a system of partial differential equations characterizing the compactification of heterotic superstrings with torsion. The bosonic fields of the ten-dimensional supergravity which arises as low energy effective theory of the 
heterotic string are the spacetime metric $g$, the NS three-form field strength (flux) $H$, the dilaton $\phi$ and the gauge connection $A$ with curvature 2-form $\Omega^{A}$. The bosonic geometry is of the form $\mathbb{R}^{1,9-d} \times X^{d}$, where the bosonic fields are non-trivial only on $X^{d}, d \leq 8$. We consider the connection $\nabla^{+}=\nabla^{g}+\frac{1}{2} H$, where $\nabla^{g}$ is the LeviCivita connection of the Riemannian metric $g$. This connection preserves the metric, $\nabla^{+} g=0$, and has totally skew-symmetric torsion $H$.

A heterotic geometry preserves supersymmetry if and only if in ten dimensions there exists at least one Majorana-Weyl spinor $\epsilon$ such that the following Killing spinor equations hold $[9,39]$

$$
\nabla^{+} \epsilon=0, \quad\left(d \phi-\frac{1}{2} H\right) \cdot \epsilon=0, \quad \Omega^{A} \cdot \epsilon=0,
$$

where $\cdot$ means Clifford action of forms on spinors.

The system of Killing spinor equations (2.1) together with the anomaly cancellation condition (1.1) is known as the Strominger system [39].

In dimension $d=6$, Strominger shows in [39] that the first two equations in (2.1) imply the 6-manifold $X$ is a compact conformally balanced Hermitian manifold with holomorphically trivial canonical bundle. Indeed, the first equation means that the holonomy group of the connection $\nabla^{+}$is contained in SU(3), i.e., the manifold $X$ admits a $\nabla^{+}$-parallel almost Hermitian structure $(g, J)$ with a nowhere vanishing $\nabla^{+}$. parallel $(3,0)$-form $\Psi_{0}$, and the second equation forces the almost complex structure $J$ to be integrable, the three-form $H=-d F(J ., J ., J)=.J d F$, where $F$ is the Kähler 2-form, $F=g(., J$.$) , and the Lee form \theta$ to be exact, $\theta=\delta F \circ J=2 d \phi$. Moreover, it is easy to see that the (3,0)-form $\Psi=e^{-2 \phi} \Psi_{0}$ is closed, and therefore holomorphic, and the metric $\bar{g}=e^{-2 \phi} g$ is balanced, i.e., its Lee form vanishes which is equivalent to the Kähler form $\bar{F}$ to be co-closed, $\delta \bar{F}=0$ (see [32] for properties and examples of balanced Hermitian manifolds).

The last equation in (2.1) is the instanton condition which means that the curvature $\Omega^{A}$ is contained in the Lie algebra $\mathfrak{s u ( 3 )}$ of the Lie group SU(3), i.e., $\Omega^{A} \wedge F^{2}=0$ and $\left(\Omega^{A}\right)^{0,2}=\left(\Omega^{A}\right)^{2,0}=0$, which is the well-known Donaldson-Uhlenbeck-Yau instanton.

Thus, let $X$ be a (compact) complex 3 -fold with holomorphically trivial canonical bundle and fix a nowhere vanishing holomorphic $(3,0)$-form $\Psi$. Let $F$ be a Hermitian metric on $X$ and denote by $\|\Psi\|_{F}$ the norm of $\Psi$ with respect to the metric $F$. Li and Yau showed in [30] that the first two equations in (2.1) are equivalent to single equation $d\left(\|\Psi\|_{F} \cdot F^{2}\right)=0$. Let $(E, h, A)$ be a holomorphic hermitian vector bundle over $X$ with curvature 2-form $\Omega^{A}$. According to [30], the Strominger system can be written as follows:

$$
\begin{aligned}
& d\left(\|\Psi\|_{F} \cdot F^{2}\right)=0, \\
& \Omega^{A} \wedge F^{2}=0, \quad\left(\Omega^{A}\right)^{0,2}=\left(\Omega^{A}\right)^{2,0}=0, \\
& d(J d F)=\frac{\alpha^{\prime}}{4}\left(\operatorname{tr} \Omega \wedge \Omega-\operatorname{tr} \Omega^{A} \wedge \Omega^{A}\right) .
\end{aligned}
$$


The connection $\nabla^{+}$with totally skew-symmetric torsion preserving the Hermitian structure $(g, J, F)$ is unique determined by the torsion 3-form $T=H=J d F=$ $-d F(J ., J ., J$.$) . This connection was used by Bismut to prove a local index formula$ for the Dolbeault operator on non-Kähler Hermitian manifolds [10] which was applied in string theory [8]. This remarkable connection is known as the Strominger-Bismut connection.

We introduce the following definition.

Definition 2.1 We will say that a compact complex manifold $X$ has the Strominger property if $X$ admits a solution to (2.2), that is to say, $X$ has holomorphically trivial canonical bundle admitting a conformally balanced Hermitian metric and an instanton bundle $E$ solving the anomaly cancellation equation (1.1).

In this definition, we refer to any solution of the Strominger system, that is, we do not restrict the connection or the instanton in the anomaly cancellation equation. However, for the solutions that we will construct in the paper it will be enough to consider the Strominger-Bismut connection, i.e., $\Omega$ in (1.1) will be the curvature of $\nabla=\nabla^{+}$. The trivial instanton $A=0$ will be enough in our study of the Strominger property for the case $\alpha^{\prime}>0$ (but not for negative $\alpha^{\prime}$, see Sect. 5).

Our aim in this paper is to study the Strominger property under holomorphic deformations of the complex structure. We will use the following terminology. Let $\Delta$ be an open disc, that we can suppose around the origin, in $\mathbb{C}$. Following [36, Definition 1.12], a given property $\mathcal{P}$ of a compact complex manifold is said to be open (or stable) under holomorphic deformations if for every holomorphic family of compact complex manifolds $\left(X_{\mathbf{t}}\right)_{\mathbf{t} \in \Delta}$ and for every $\mathbf{t}_{0} \in \Delta$, the following implication holds:

$X_{\mathbf{t}_{0}}$ has the property $\mathcal{P} \Longrightarrow X_{\mathbf{t}}$ has the property $\mathcal{P}$ for all $\mathbf{t} \in \Delta$ sufficiently close to $\mathbf{t}_{0}$.

A given property $\mathcal{P}$ of a compact complex manifold is said to be closed under holomorphic deformations, if for every holomorphic family of compact complex manifolds $\left(X_{\mathbf{t}}\right)_{\mathbf{t} \in \Delta}$ and for every $\mathbf{t}_{0} \in \Delta$ the following implication holds:

$X_{\mathbf{t}}$ has the property $\mathcal{P}$ for all $\mathbf{t} \in \Delta-\left\{\mathbf{t}_{0}\right\} \Longrightarrow X_{\mathbf{t}_{0}}$ has the property $\mathcal{P}$.

In [1] and [13] it is proved, respectively, that the balanced property is neither open nor closed (in the balanced class). Roughly speaking, what we will prove in the next sections is that the balanced property is neither open nor closed even in the Strominger class, both in the case of positive slope parameter as well as in the case of negative slope parameter. This clearly implies the non-openness and the non-closedness of the Strominger property under holomorphic deformations.

\section{The Strominger Property with Positive $\alpha^{\prime}$ is Not Stable}

In this section, we show that the Strominger property with positive slope parameter $\alpha^{\prime}$ in (1.1) is not stable by small deformations of the complex structure. We will prove a stronger result, namely that a small holomorphic deformation of a compact complex manifold satisfying the Strominger system with $\alpha^{\prime}>0$ may not admit any balanced metric.

We will focus on the complex geometry of the (real) manifold underlying the Iwasawa manifold. Let us denote by $M$ this 6-dimensional compact manifold. It is 
well known that $M$ is a nilmanifold, i.e., it is a compact manifold $M=\Gamma \backslash G$ obtained as a quotient of a simply connected nilpotent Lie group $G$ by a lattice $\Gamma$ of maximal rank. The Lie algebra of $G$ is isomorphic to $\mathfrak{h}_{5}=(0,0,0,0,13+42,14+23)$ (in the notation of [38]).

We consider invariant complex structures $J$ on $M$, that is to say, $J$ is a complex structure coming from a left-invariant complex structure on $G$ by passing to the quotient. By [38], the canonical bundle of any $(M, J)$ is holomorphically trivial. To our knowledge, it is an open question if the Iwasawa manifold, i.e., $M$ with its standard complex parallelizable structure, can admit or not any solution to the Strominger system with $\alpha^{\prime}>0$ (see Sect. 5 for more details). Next we look for appropriate invariant complex structures on $M$.

There are many invariant complex structures on $M$, but in order to prove the nonstability of the balanced property for solutions of the Strominger system with $\alpha^{\prime}>0$, we need to find an invariant complex structure $J$ on $M$ admitting such solutions and for which the dimension of the Bott-Chern cohomology group $H_{\mathrm{BC}}^{2,2}(M, J)$ does not attain a minimum in the space of all invariant complex structures on $M$. Indeed, such a choice is necessary for performing later an appropriate holomorphic deformation, since by [5, Proposition 4.1] if $\left\{X_{t}\right\}_{t \in(-\varepsilon, \varepsilon)}, \varepsilon>0$, is any differentiable family of deformations of a compact balanced manifold $X_{0}$ of complex dimension $n$ such that the upper-semi-continuous function $t \mapsto \operatorname{dim}_{\mathbb{C}} H_{\mathrm{BC}}^{n-1, n-1}\left(X_{t}\right)$ is constant, then $X_{t}$ also admits a balanced metric for any $t$ close enough to 0 .

From [29, Appendix] and [6, Table 2], it follows that the minimum value of the dimension of the Bott-Chern cohomology group $H_{\mathrm{BC}}^{2,2}(M, J)$ in the space of invariant complex structures $J$ on the manifold $M$ is equal to 6. By [37, Theorem 2.6], the complex structure of any sufficiently small deformation of $(M, J)$ is again invariant. So, if $\left(X_{\mathbf{t}}\right)_{\mathbf{t} \in \Delta}$ is any (sufficiently small) holomorphic deformation of a complex manifold $X_{0}=(M, J)$ such that $\operatorname{dim}_{\mathbb{C}} H_{\mathrm{BC}}^{2,2}(M, J)=6$, then the function $\mathbf{t} \mapsto \operatorname{dim}_{\mathbb{C}} H_{\mathrm{BC}}^{2,2}\left(X_{\mathbf{t}}\right)$ is constant.

In conclusion, we need to find a complex structure admitting balanced metrics for which the dimension of its Bott-Chern cohomology group of bidegree $(2,2)$ is at least 7. But such complex structures are already classified in [29, Table 1]. In fact, up to isomorphism of the complex structure (and apart from the Iwasawa manifold), we are led to consider a complex structure $J^{s}$ in the following family:

$$
d \omega^{1}=d \omega^{2}=0, \quad d \omega^{3}=\omega^{12}+\omega^{1 \overline{1}}-s^{2} \omega^{2 \overline{2}}, \quad s \in(0,1 / 2) .
$$

From now on, given any $s \in(0,1 / 2)$, we will consider the compact complex manifold $X^{s}=\left(M, J^{s}\right)$ of complex dimension 3, where $M$ is the (real) nilmanifold underlying the Iwasawa manifold and $J^{s}$ is the (integrable almost) complex structure defined by the (1,0)-basis $\left\{\omega^{1}, \omega^{2}, \omega^{3}\right\}$ satisfying Eq. (3.1). Here $\omega^{j k}$, resp. $\omega^{j \bar{k}}$, means the wedge product $\omega^{j} \wedge \omega^{k}$, resp. $\omega^{j} \wedge \omega^{\bar{k}}$, where $\omega^{\bar{k}}$ indicates the complex conjugate of $\omega^{k}$, and we shall use a similar abbreviated notation for forms of other bidegrees.

In the following result, we prove that any compact complex manifold $X^{s}=\left(M, J^{s}\right)$ has the Strominger property with positive slope parameter in the anomaly cancellation equation. 
Proposition 3.1 For every $s \in\left(0, \frac{1}{2}\right)$, the compact complex manifold $X^{s}=\left(M, J^{s}\right)$ admits a solution to the Strominger system with $\alpha^{\prime}>0$.

Proof Firstly, notice that it follows directly from (3.1) that the (3,0)-form $\Psi_{s}=s \omega^{123}$ is holomorphic, so $X^{s}$ has holomorphically trivial canonical bundle.

Let us consider the $J^{s}$-Hermitian metric $F_{S}$ on $X^{s}$ given by

$$
F_{s}=\frac{i}{2}\left(\omega^{1 \overline{1}}+s^{2} \omega^{2 \overline{2}}+\omega^{3 \overline{3}}\right) .
$$

Using the structure equations given in Eq. (3.1), we have

$$
\begin{aligned}
-4 d F_{s} \wedge F_{s} & =d \omega^{3 \overline{3}} \wedge\left(\omega^{1 \overline{1}}+s^{2} \omega^{2 \overline{2}}+\omega^{3 \overline{3}}\right) \\
& =\left(\omega^{12 \overline{3}}+\omega^{1 \overline{1} \overline{3}}-s^{2} \omega^{2 \overline{2} \overline{3}}+\omega^{1 \overline{1} 3}-s^{2} \omega^{2 \overline{2} 3}-\omega^{3 \overline{1} \overline{2}}\right) \wedge\left(\omega^{1 \overline{1}}+s^{2} \omega^{2 \overline{2}}\right) \\
& =\left(\omega^{1 \overline{1} \overline{3}}+\omega^{1 \overline{1} 3}\right) \wedge\left(s^{2} \omega^{2 \overline{2}}\right)-s^{2}\left(\omega^{2 \overline{2} \overline{3}}+\omega^{2 \overline{2} 3}\right) \wedge \omega^{1 \overline{1}} \\
& =0 .
\end{aligned}
$$

Hence, $d F_{s}^{2}=0$, i.e., the metric $F_{s}$ is a balanced metric on $X^{s}$.

The torsion 3-form $T_{s}=J^{s} d F_{s}$ is given by

$$
T_{s}=-\frac{1}{2}\left(\omega^{12 \overline{3}}-\omega^{1 \overline{1} \overline{3}}+s^{2} \omega^{2 \overline{2} \overline{3}}+\omega^{1 \overline{1} 3}-s^{2} \omega^{2 \overline{2} 3}+\omega^{3 \overline{1} \overline{2}}\right) .
$$

By (3.1) we get

$$
d T_{s}=d J^{s} d F_{s}=\frac{2 s^{2}+1}{s^{4}} \omega^{1 \overline{1} 2 \overline{2}} .
$$

We will consider the Strominger-Bismut connection in the anomaly cancellation condition. The curvature forms can be obtained more easily in a basis adapted to the $\mathrm{SU}(3)$-structure $\left(F_{s}, \Psi_{s}\right)$. Let $\left\{e^{k}\right\}_{k=1}^{6}$ be the basis of real 1 -forms defined as

$$
\begin{aligned}
& e^{1}=\mathfrak{R e} \omega^{1}, e^{2}=\mathfrak{I m} \omega^{1}, e^{3}=\mathfrak{R e}\left(s \omega^{2}\right), \\
& e^{4}=\mathfrak{I m}\left(s \omega^{2}\right), \quad e^{5}=\mathfrak{R e} \omega^{3}, \quad e^{6}=\mathfrak{I m} \omega^{3},
\end{aligned}
$$

that is, $\omega^{1}=e^{1}+i e^{2}, s \omega^{2}=e^{3}+i e^{4}$, and $\omega^{3}=e^{5}+i e^{6}$. Hence, the complex structure $J^{s}$ and the $\mathrm{SU}(3)$-structure $\left(F_{s}, \Psi_{s}\right)$ express as

$$
\begin{aligned}
& J^{s} e^{1}=-e^{2}, J^{s} e^{3}=-e^{4}, J^{s} e^{5}=-e^{6}, \\
& F_{s}=e^{12}+e^{34}+e^{56}, \\
& \Psi_{s}=\left(e^{1}+i e^{2}\right) \wedge\left(e^{3}+i e^{4}\right) \wedge\left(e^{5}+i e^{6}\right) .
\end{aligned}
$$

In this adapted basis, the curvature 2-forms of the Strominger-Bismut connection, which for simplicity we will denote by $\Omega_{j}^{i}$ instead of $\left(\Omega_{s}\right)_{j}^{i}$, are

$$
\Omega_{2}^{1}=-4\left(e^{12}-e^{34}\right)+\frac{2}{s}\left(e^{14}+e^{23}\right)+\frac{2}{s^{2}} e^{34},
$$




$$
\begin{aligned}
& \Omega_{3}^{1}=\Omega_{4}^{2}=-\frac{1}{s^{2}}\left(e^{13}+e^{24}\right), \\
& \Omega_{4}^{1}=-\Omega_{3}^{2}=-\frac{1}{s^{2}}\left(e^{14}-e^{23}\right), \\
& \Omega_{5}^{1}=\Omega_{6}^{2}=-\frac{2}{s} e^{46}, \\
& \Omega_{6}^{1}=-\Omega_{5}^{2}=\frac{2}{s} e^{36}, \\
& \Omega_{4}^{3}=4\left(e^{12}-e^{34}\right)-\frac{2}{s}\left(e^{14}+e^{23}\right)+\frac{2}{s^{2}} e^{12}, \\
& \Omega_{5}^{3}=\Omega_{6}^{4}=-\frac{2}{s} e^{26}, \\
& \Omega_{6}^{3}=-\Omega_{5}^{4}=\frac{2}{s} e^{16}, \\
& \Omega_{6}^{5}=-\Omega_{2}^{1}-\Omega_{4}^{3}=-\frac{2}{s^{2}}\left(e^{12}+e^{34}\right) .
\end{aligned}
$$

Now, a direct calculation shows that

$$
\operatorname{tr} \Omega_{s} \wedge \Omega_{s}=\sum_{1 \leq i<j \leq 6} \Omega_{j}^{i} \wedge \Omega_{j}^{i}=-16 \frac{4 s^{2}+1}{s^{6}} e^{1234}=4 \frac{4 s^{2}+1}{s^{4}} \omega^{1 \overline{1} 2 \overline{2}} .
$$

Taking $A=0$ (see Remark 5.2 for solutions with non-flat instanton), from the previous equality and (3.3), we get

$$
\frac{2 s^{2}+1}{s^{4}} \omega^{1 \overline{1} 2 \overline{2}}=d T_{s}=\frac{\alpha^{\prime}}{4}\left(\operatorname{tr} \Omega_{s} \wedge \Omega_{s}-\operatorname{tr} \Omega^{A} \wedge \Omega^{A}\right)=4 \alpha^{\prime} \frac{4 s^{2}+1}{s^{4}} \omega^{1 \overline{1} 2 \overline{2}} .
$$

Therefore, the anomaly cancellation equation is solved for positive $\alpha^{\prime}=\frac{2 s^{2}+1}{4\left(4 s^{2}+1\right)}$.

Next we want to perform an appropriate holomorphic deformation of the compact complex manifold $X^{s}=\left(M, J^{s}\right)$. As we recalled above, since $J^{s}$ is an invariant complex structure, by [37, Theorem 2.6] any sufficiently small deformation of $J^{s}$ is given again by an invariant complex structure on the nilmanifold $M$. Notice also that the Dolbeault cohomology of $X^{s}$ is isomorphic to the Lie algebra cohomology, in particular

$$
H^{0,1}\left(X^{s}\right) \cong H^{0,1}\left(\mathfrak{h}_{5}, J^{s}\right)=\left\langle\left[\omega^{\overline{1}}\right],\left[\omega^{\overline{2}}\right]\right\rangle .
$$

Following an idea in [31, Example 8], we first consider invariant almost complex structures $J_{\Phi}^{S}$, which are "near" to $J^{s}$, defined by the following basis of $(1,0)$-forms

$$
\left\{\begin{array}{l}
\omega_{\Phi}^{1}=\omega^{1}+\Phi_{1}^{1} \omega^{\overline{1}}+\Phi_{2}^{1} \omega^{\overline{2}}, \\
\omega_{\Phi}^{2}=\omega^{2}+\Phi_{1}^{2} \omega^{\overline{1}}+\Phi_{2}^{2} \omega^{\overline{2}}, \\
\omega_{\Phi}^{3}=\omega^{3},
\end{array}\right.
$$


where the coefficients $\Phi_{1}^{1}, \Phi_{2}^{1}, \Phi_{1}^{2}, \Phi_{2}^{2} \in \mathbb{C}$. The integrability of the almost complex structure $J_{\Phi}^{s}$ is equivalent to the closedness of the $(3,0)$-form

$$
\begin{aligned}
\omega_{\Phi}^{123}= & \omega^{123}+\left(\Phi_{1}^{2} \omega^{1 \overline{1}}+\Phi_{2}^{2} \omega^{1 \overline{2}}-\Phi_{1}^{1} \omega^{2 \overline{1}}-\Phi_{2}^{1} \omega^{2 \overline{2}}\right) \wedge \omega^{3} \\
& +\left(\Phi_{1}^{1} \Phi_{2}^{2}-\Phi_{2}^{1} \Phi_{1}^{2}\right) \omega^{\overline{1} \overline{2} 3} .
\end{aligned}
$$

A direct calculation using (3.1) and (3.4) shows that $J_{\Phi}^{s}$ is integrable if and only if

$$
\Phi_{1}^{1} \Phi_{2}^{2}-\Phi_{2}^{1} \Phi_{1}^{2}+\Phi_{2}^{1}+s^{2} \Phi_{1}^{2}=0 .
$$

In the following result, we prove the existence of a direction in the deformation space such that the resulting compact complex manifolds do not admit any solution to the Strominger system.

Theorem 3.2 There exists a holomorphic family of compact complex manifolds $\left(X_{\mathbf{t}}\right)_{\mathbf{t} \in \Delta}$ of complex dimension 3 , where $\Delta=\{\mathbf{t} \in \mathbb{C}|| \mathbf{t} \mid<\varepsilon\}, \varepsilon>0$, such that $X_{\mathbf{t}}$ has holomorphically trivial canonical bundle for any $\mathbf{t} \in \Delta$, and

(i) $X_{0}$ admits a solution to the Strominger system with $\alpha^{\prime}>0$, but

(ii) $X_{\mathbf{t}}$ does not admit balanced metrics for any $\mathbf{t} \in \Delta-\{\mathfrak{I m} \mathbf{t}=0\}$.

In particular, the Strominger property is not stable under small deformations of the complex structure.

Proof Let $s \in(0,1 / 2)$ and consider $X=X^{s}$, where $X^{s}$ is the compact complex manifold constructed above. By Proposition 3.1, we know that $X$ has the Strominger property with $\alpha^{\prime}>0$. We will construct a small deformation $X_{\mathbf{t}}$ of $X_{0}=X$ for which there are non-zero $\mathbf{t}$ 's arbitrary close to 0 such that the compact complex manifold $X_{\mathbf{t}}$ does not admit any balanced metric.

To construct such a deformation, we first choose $\Phi_{1}^{1}=\Phi_{2}^{2}=0$ in (3.4). Now, the integrability condition (3.5) is equivalent to $\Phi_{1}^{2}\left(\Phi_{2}^{1}-s^{2}\right)-\Phi_{2}^{1}=0$, where $\Phi_{2}^{1}, \Phi_{1}^{2} \in \mathbb{C}$. We consider the open disc $\Delta=\Delta\left(0, s^{2}\right)$ around $0 \in \mathbb{C}$ and of radius $s^{2}$, and we take $\Phi_{2}^{1} \in \Delta$.

From now on, we denote $\Phi_{2}^{1}=\mathbf{t}$. Since $\mathbf{t} \in \Delta\left(0, s^{2}\right) \subset \mathbb{C}$, we have $\mathbf{t}-s^{2} \neq 0$ and the integrability condition (3.5) reduces to $\Phi_{1}^{2}=\mathbf{t} /\left(\mathbf{t}-s^{2}\right)$. So, the system (3.4) is written as follows:

$$
\omega_{\mathbf{t}}^{1}=\omega^{1}+\mathbf{t} \omega^{\overline{2}}, \quad \omega_{\mathbf{t}}^{2}=\omega^{2}+\frac{\mathbf{t}}{\mathbf{t}-s^{2}} \omega^{\overline{1}}, \quad \omega_{\mathbf{t}}^{3}=\omega^{3}, \quad \text { for } \mathbf{t} \in \Delta\left(0, s^{2}\right) .
$$

Hence, we have, for each $\mathbf{t} \in \Delta\left(0, s^{2}\right)$, an invariant complex structure $J_{\mathbf{t}}$ defined by this basis of bidegree $(1,0)$ on the real nilmanifold $M$. We denote by $X_{\mathbf{t}}$ the compact complex manifold $\left(M, J_{\mathbf{t}}\right)$.

Notice that since the Dolbeault cohomology group $H^{0,1}\left(X_{0}\right)=\left\langle\left[\omega^{\overline{1}}\right],\left[\omega^{\overline{2}}\right]\right\rangle$, one can consider $X_{\mathbf{t}}$ as a small deformation of the compact complex manifold $X_{0}$ given by

$$
\frac{\mathbf{t}}{\mathbf{t}-s^{2}} \frac{\partial}{\partial z_{2}} \otimes \omega^{\overline{1}}+\mathbf{t} \frac{\partial}{\partial z_{1}} \otimes \omega^{\overline{2}} \in H^{0,1}\left(X_{0}, T^{1,0} X_{0}\right)
$$


Now, using (3.1), the complex structure equations of $X_{\mathbf{t}}$ are

$$
d \omega_{\mathbf{t}}^{1}=d \omega_{\mathbf{t}}^{2}=0, \quad d \omega_{\mathbf{t}}^{3}=a(\mathbf{t}) \omega_{\mathbf{t}}^{12}+b(\mathbf{t}) \omega_{\mathbf{t}}^{1 \overline{1}}+c(\mathbf{t}) \omega_{\mathbf{t}}^{2 \overline{2}},
$$

where

$$
\begin{aligned}
& a(\mathbf{t})=\left(\mathbf{t}-s^{2}\right) \frac{\overline{\mathbf{t}}(1-\overline{\mathbf{t}})-s^{2}}{\left.|\mathbf{t}-| \mathbf{t}\right|^{2}-\left.s^{2}\right|^{2}}, \quad b(\mathbf{t})=-s^{2} \frac{\overline{\mathbf{t}}(1-\mathbf{t})-s^{2}}{\left.|\mathbf{t}-| \mathbf{t}\right|^{2}-\left.s^{2}\right|^{2}}, \\
& c(\mathbf{t})=\left|\mathbf{t}-s^{2}\right|^{2} \frac{\mathbf{t}(1-\overline{\mathbf{t}})-s^{2}}{\left.|\mathbf{t}-| \mathbf{t}\right|^{2}-\left.s^{2}\right|^{2}} .
\end{aligned}
$$

Notice that $a(0)=b(0)=1$ and $c(0)=-s^{2}$, so the coefficients are non-zero for sufficiently small $\mathbf{t}$. Furthermore, a direct calculation shows that $\mathbf{t} \neq|\mathbf{t}|^{2}+s^{2}$ and $a(\mathbf{t}), b(\mathbf{t}), c(\mathbf{t})$ do not vanish for any $\mathbf{t} \in \Delta\left(0, s^{2}\right)$.

Let us consider the new basis $\left\{\eta_{\mathbf{t}}^{1}, \eta_{\mathbf{t}}^{2}, \eta_{\mathbf{t}}^{3}\right\}$ of bidegree $(1,0)$ for $X_{\mathbf{t}}$ defined by

$$
\eta_{\mathbf{t}}^{1}=a(\mathbf{t}) \omega_{\mathbf{t}}^{1}, \quad \eta_{\mathbf{t}}^{2}=\frac{|a(\mathbf{t})|^{2}}{b(\mathbf{t})} \omega_{\mathbf{t}}^{2}, \quad \eta_{\mathbf{t}}^{3}=\frac{|a(\mathbf{t})|^{2}}{b(\mathbf{t})} \omega_{\mathbf{t}}^{3} .
$$

This basis satisfies

$$
d \eta_{\mathbf{t}}^{1}=d \eta_{\mathbf{t}}^{2}=0, \quad d \eta_{\mathbf{t}}^{3}=\eta_{\mathbf{t}}^{12}+\eta_{\mathbf{t}}^{1 \overline{1}}+D(\mathbf{t}) \eta_{\mathbf{t}}^{2 \overline{2}}
$$

where

$$
D(\mathbf{t})=\frac{\overline{b(\mathbf{t})} c(\mathbf{t})}{|a(\mathbf{t})|^{2}}=-s^{2} \frac{\left(\mathbf{t}-|\mathbf{t}|^{2}-s^{2}\right)^{2}}{\left|\mathbf{t}-\mathbf{t}^{2}-s^{2}\right|^{2}} .
$$

Since every $X_{\mathbf{t}}$ is a nilmanifold endowed with an invariant complex structure, by symmetrization [22], the compact complex manifold $X_{\mathbf{t}}$ has a balanced metric if and only if it has an invariant one. Now, by [42, Proposition 2.3] any invariant Hermitian metric $F$ on $X_{\mathbf{t}}$ can be written as

$$
2 F=i\left(\eta_{\mathbf{t}}^{1 \overline{1}}+p^{2} \eta_{\mathbf{t}}^{2 \overline{2}}+q^{2} \eta_{\mathbf{t}}^{3 \overline{3}}\right)+u \eta_{\mathbf{t}}^{1 \overline{2}}-\bar{u} \eta_{\mathbf{t}}^{2 \overline{1}}
$$

for some $p, q \in \mathbb{R}^{*}, u \in \mathbb{C}$ with $p^{2}>|u|^{2}$. Moreover, the metric $F$ is balanced if and only if

$$
p^{2}+D(\mathbf{t})=0
$$

Next we prove that the previous condition does not hold for any $\mathbf{t} \in \Delta\left(0, s^{2}\right)-\{\mathfrak{I m} \mathbf{t}=$ 0 \}. Indeed, a direct calculation shows that $D(\mathbf{t})$ is real if and only if

$$
t_{2}\left(t_{1}^{2}+t_{2}^{2}-t_{1}+s^{2}\right)=0
$$


where $t_{1}=\mathfrak{R e}(\mathbf{t})$ and $t_{2}=\mathfrak{I m}(\mathbf{t})$, i.e., $\mathbf{t}=t_{1}+i t_{2}$. It is easy to see that $t_{1}^{2}+t_{2}^{2}-$ $t_{1}+s^{2} \neq 0$ for any $\mathbf{t} \in \Delta\left(0, s^{2}\right)$, so the condition (3.7) is satisfied if and only if $t_{2}=0$. Therefore, for any $\mathbf{t} \in \Delta\left(0, s^{2}\right)-\left\{t_{2}=0\right\}$ we have that $\mathfrak{I m} D(\mathbf{t}) \neq 0$ and the balanced condition is not satisfied.

\section{The Strominger Property with Positive $\alpha^{\prime}$ is Not Closed}

Our aim in this section is to prove that the Strominger property with positive slope parameter is not closed under holomorphic deformations.

We will focus on the complex geometry of the nilmanifold corresponding to the nilpotent Lie algebra $\mathfrak{h}_{4}=(0,0,0,0,12,14+23)$ in the notation of [38]. This 6-dimensional compact manifold, which we will denote by $N$, has many invariant complex structures $J$. By [38], the canonical bundle of any $(N, J)$ is holomorphically trivial.

Let $J_{0}$ be the invariant complex structure on $N$ defined by a (1,0)-basis $\left\{\eta^{1}, \eta^{2}, \eta^{3}\right\}$ satisfying the equations

$$
d \eta^{1}=d \eta^{2}=0, \quad d \eta^{3}=\frac{i}{2} \eta^{1 \overline{1}}+\frac{1}{2} \eta^{1 \overline{2}}+\frac{1}{2} \eta^{2 \overline{1}} .
$$

Maclaughlin, Pedersen, Poon, and Salamon studied in [31, Example 8] the deformation parameter space of $J_{0}$. Any invariant complex structure sufficiently near to $J_{0}$ has a basis of $(1,0)$-forms $\left\{\eta_{\Phi}^{1}, \eta_{\Phi}^{2}, \eta_{\Phi}^{3}\right\}$ that can be written as

$$
\left\{\begin{array}{l}
\eta_{\Phi}^{1}=\eta^{1}+\Phi_{1}^{1} \eta^{\overline{1}}+\Phi_{2}^{1} \eta^{\overline{2}} \\
\eta_{\Phi}^{2}=\eta^{2}+\Phi_{1}^{2} \eta^{\overline{1}}+\Phi_{2}^{2} \eta^{\overline{2}} \\
\eta_{\Phi}^{3}=\eta^{3}+\Phi_{3}^{3} \eta^{\overline{3}}
\end{array}\right.
$$

where the coefficients $\Phi_{1}^{1}, \Phi_{2}^{1}, \Phi_{1}^{2}, \Phi_{2}^{2}, \Phi_{3}^{3} \in \mathbb{C}$ are sufficiently small, and they satisfy the integrability condition. Notice that the latter is equivalent to the closedness of the $(3,0)$-form $\eta_{\Phi}^{123}$, and using (4.1) it is given by

$$
i\left(1+\Phi_{3}^{3}\right) \Phi_{2}^{1}=\left(1-\Phi_{3}^{3}\right)\left(\Phi_{1}^{1}-\Phi_{2}^{2}\right)
$$

We will consider a holomorphic family constructed in [13] to answer a question in [36] about the closedness of the balanced property. Let us take $\Phi_{1}^{1}=\mathbf{t}, \Phi_{2}^{1}=-i \mathbf{t}$ and $\Phi_{1}^{2}=\Phi_{2}^{2}=\Phi_{3}^{3}=0$ in the parameter space (4.2), where $\mathbf{t} \in \Delta=\Delta(0,1)=$ $\{\mathbf{t} \in \mathbb{C}|| \mathbf{t} \mid<1\}$. That is to say, for each $\mathbf{t} \in \Delta$, we consider the basis $\left\{\eta_{\mathbf{t}}^{1}, \eta_{\mathbf{t}}^{2}, \eta_{\mathbf{t}}^{3}\right\}$ of $(1,0)$-forms given by

$$
\eta_{\mathbf{t}}^{1}=\eta^{1}+\mathbf{t} \eta^{\overline{1}}-i \mathbf{t} \eta^{\overline{2}}, \quad \eta_{\mathbf{t}}^{2}=\eta^{2}, \quad \eta_{\mathbf{t}}^{3}=\eta^{3}
$$

This basis defines an invariant complex structure $J_{\mathbf{t}}$ on the nilmanifold $N$, and we denote by $X_{\mathbf{t}}$ the corresponding compact complex manifold $\left(N, J_{\mathbf{t}}\right)$. 
In [13, Theorem 5.9], it is proved that a compact complex manifold in the holomorphic family $\left(X_{\mathbf{t}}\right)_{\mathbf{t} \in \Delta}$ has a balanced metric if and only if $\mathbf{t} \in \Delta-\{0\}$. Thus, there does not exist any balanced metric on the central limit $X_{0}=\left(N, J_{0}\right)$, and the balanced property is not closed by holomorphic deformations.

In the following result, we prove that any compact complex manifold $X_{\mathbf{t}}, \mathbf{t} \neq 0$, admits in addition a solution to the Strominger system with positive slope parameter $\alpha^{\prime}$.

Theorem 4.1 There exists a holomorphic family of compact complex manifolds $\left(X_{\mathbf{t}}\right)_{\mathbf{t} \in \Delta}$, where $\Delta=\{\mathbf{t} \in \mathbb{C}|| \mathbf{t} \mid<1\}$, such that $X_{\mathbf{t}}$ has holomorphically trivial canonical bundle for any $\mathbf{t} \in \Delta$, and

(i) $X_{\mathbf{t}}$ admits a solution to the Strominger system with $\alpha^{\prime}>0$ for every $\mathbf{t} \in \Delta-\{0\}$,

(ii) $X_{0}$ does not admit any balanced Hermitian metric.

In particular, the Strominger property with positive slope parameter is not closed under holomorphic deformations.

Proof A direct calculation using (4.1) shows that the complex structure equations for $X_{\mathbf{t}}$, with respect to the basis (4.4), are

$$
d \eta_{\mathbf{t}}^{1}=d \eta_{\mathbf{t}}^{2}=0, \quad 2\left(1-|\mathbf{t}|^{2}\right) d \eta_{\mathbf{t}}^{3}=2 \overline{\mathbf{t}} \eta_{\mathbf{t}}^{12}+i \eta_{\mathbf{t}}^{1 \overline{1}}+\eta_{\mathbf{t}}^{1 \overline{2}}+\eta_{\mathbf{t}}^{2 \overline{1}}-i|\mathbf{t}|^{2} \eta_{\mathbf{t}}^{2 \overline{2}},
$$

for each $\mathbf{t} \in \Delta$.

By [13, Theorem 5.9], the compact complex manifold $X_{0}$ does not admit any balanced metric. For each $\mathbf{t} \in \Delta-\{0\}$, we consider the Hermitian metric $F_{\mathbf{t}, r}$ on $X_{\mathbf{t}}$ given by

$$
F_{\mathbf{t}, r}=\frac{i}{2}\left(\eta_{\mathbf{t}}^{1 \overline{1}}+|\mathbf{t}|^{2} \eta_{\mathbf{t}}^{2 \overline{2}}+r^{2} \eta_{\mathbf{t}}^{3 \overline{3}}\right)
$$

where $r \in \mathbb{R}^{*}$. Now,

$$
\begin{aligned}
-4 d F_{\mathbf{t}, r} \wedge F_{\mathbf{t}, r} & =r^{2} d \eta_{\mathbf{t}}^{3 \overline{3}} \wedge\left(\eta_{\mathbf{t}}^{1 \overline{1}}+|\mathbf{t}|^{2} \eta_{\mathbf{t}}^{2 \overline{2}}+r^{2} \eta_{\mathbf{t}}^{3 \overline{3}}\right) \\
& =r^{2}\left(d \eta_{\mathbf{t}}^{3} \wedge \eta_{\mathbf{t}}^{\overline{3}}-\eta_{\mathbf{t}}^{3} \wedge d \eta_{\mathbf{t}}^{\overline{3}}\right) \wedge\left(\eta_{\mathbf{t}}^{1 \overline{1}}+|\mathbf{t}|^{2} \eta_{\mathbf{t}}^{2 \overline{2}}\right) \\
& =0
\end{aligned}
$$

because $d \eta_{\mathbf{t}}^{3} \wedge\left(\eta_{\mathbf{t}}^{1 \overline{1}}+|\mathbf{t}|^{2} \eta_{\mathbf{t}}^{2 \overline{2}}\right)=0$ by (4.5). Hence, the metric $F_{\mathbf{t}, r}$ is a balanced metric on $X_{\mathbf{t}}$ for any $0<|\mathbf{t}|<1$ and $r \in \mathbb{R}^{*}$.

For each $\mathbf{t} \in \Delta-\{0\}$, we consider the real basis of 1 -forms $\left\{e^{1}, \ldots, e^{6}\right\}$ defined as

$$
\begin{aligned}
& e^{1}=\mathfrak{R e} \eta_{\mathbf{t}}^{1}, \quad e^{2}=\mathfrak{I m} \eta_{\mathbf{t}}^{1}, \quad e^{3}=\mathfrak{R e}\left(|\mathbf{t}| \eta_{\mathbf{t}}^{2}\right), \\
& e^{4}=\mathfrak{I m}\left(|\mathbf{t}| \eta_{\mathbf{t}}^{2}\right), \quad e^{5}=\mathfrak{R e}\left(r \eta_{\mathbf{t}}^{3}\right), \quad e^{6}=\mathfrak{I m}\left(r \eta_{\mathbf{t}}^{3}\right),
\end{aligned}
$$

that is,

$$
e^{1}+i e^{2}=\eta_{\mathbf{t}}^{1}, \quad e^{3}+i e^{4}=|\mathbf{t}| \eta_{\mathbf{t}}^{2}, \quad e^{5}+i e^{6}=r \eta_{\mathbf{t}}^{3} .
$$


Hence, the complex structure $J_{\mathbf{t}}$ and the $\operatorname{SU}(3)$-structure $\left(F_{\mathbf{t}, r}, \Psi_{\mathbf{t}, r}=r|\mathbf{t}| \eta_{\mathbf{t}}^{123}\right)$ express in this basis as

$$
\begin{aligned}
J_{\mathbf{t}} e^{1} & =-e^{2}, J_{\mathbf{t}} e^{3}=-e^{4}, J_{\mathbf{t}} e^{5}=-e^{6}, \\
F_{\mathbf{t}, r} & =e^{12}+e^{34}+e^{56} \\
\Psi_{\mathbf{t}, r} & =\left(e^{1}+i e^{2}\right) \wedge\left(e^{3}+i e^{4}\right) \wedge\left(e^{5}+i e^{6}\right) .
\end{aligned}
$$

Moreover, it follows from (4.5) that the structure equations in the adapted basis $\left\{e^{k}\right\}_{k=1}^{6}$ are

$$
\left\{\begin{array}{l}
d e^{1}=d e^{2}=d e^{3}=d e^{4}=0, \\
d e^{5}=\frac{r}{1-|\mathbf{t}|^{2}}\left(e^{12}-e^{34}\right)+\frac{r t_{1}}{|\mathbf{t}|\left(1-|\mathbf{t}|^{2}\right)}\left(e^{13}-e^{24}\right)+\frac{r t_{2}}{|\mathbf{t}|\left(1-|\mathbf{t}|^{2}\right)}\left(e^{14}+e^{23}\right), \\
d e^{6}=-\frac{r t_{2}}{|\mathbf{t}|\left(1-|\mathbf{t}|^{2}\right)}\left(e^{13}-e^{24}\right)-\frac{r\left(1-t_{1}\right)}{|\mathbf{t}|\left(1-|\mathbf{t}|^{2}\right)} e^{14}+\frac{r\left(1+t_{1}\right)}{|\mathbf{t}|\left(1-|\mathbf{t}|^{2}\right)} e^{23},
\end{array}\right.
$$

where $t_{1}=\mathfrak{R e}(\mathbf{t})$ and $t_{2}=\mathfrak{I m}(\mathbf{t})$, i.e., $\mathbf{t}=t_{1}+i t_{2}$.

Using (4.8), we get that the torsion 3-form $T_{\mathbf{t}, r}=J_{\mathbf{t}} d F_{\mathbf{t}, r}$ is given by

$$
\begin{aligned}
\frac{|\mathbf{t}|\left(1-|\mathbf{t}|^{2}\right)}{r} T_{\mathbf{t}, r}= & |\mathbf{t}| e^{125}-t_{1} e^{135}+t_{2} e^{136}-t_{2} e^{145}-\left(1+t_{1}\right) e^{146} \\
& -t_{2} e^{235}+\left(1-t_{1}\right) e^{236}+t_{1} e^{245}-t_{2} e^{246}-|\mathbf{t}| e^{345} .
\end{aligned}
$$

Again from (4.8), we get

$$
d T_{\mathbf{t}, r}=-\frac{2 r^{2}\left(1+3|\mathbf{t}|^{2}\right)}{|\mathbf{t}|^{2}\left(1-|\mathbf{t}|^{2}\right)^{2}} e^{1234}=\frac{r^{2}\left(1+3|\mathbf{t}|^{2}\right)}{2\left(1-|\mathbf{t}|^{2}\right)^{2}} \eta_{\mathbf{t}}^{1 \overline{1} 2 \overline{2}}
$$

In the last equality, we have used the relation $e^{1234}=-\frac{|\mathbf{t}|^{2}}{4} \eta_{\mathbf{t}}^{1 \overline{1} 2 \overline{2}}$.

A long but direct calculation shows that, in the adapted basis $\left\{e^{k}\right\}_{k=1}^{6}$, the curvature 2 -forms of the Strominger-Bismut connection, which for simplicity we will denote by $\Omega_{j}^{i}$ instead of $\left(\Omega_{\mathbf{t}, r}\right)_{j}^{i}$, are as follows:

$$
\begin{aligned}
& \rho(\mathbf{t}, r) \Omega_{2}^{1}=-|\mathbf{t}|^{2} e^{12}-t_{1}|\mathbf{t}|\left(e^{13}-e^{24}\right)-t_{2}|\mathbf{t}|\left(e^{14}+e^{23}\right)+3|\mathbf{t}|^{2} e^{34}, \\
& \rho(\mathbf{t}, r) \Omega_{3}^{1}=-|\mathbf{t}|^{2}\left(e^{13}+e^{24}\right)+2|\mathbf{t}| e^{56} \\
& \rho(\mathbf{t}, r) \Omega_{4}^{1}=-t_{2}\left(e^{13}-e^{24}\right)-\left(1-t_{1}+|\mathbf{t}|^{2}\right) e^{14}+\left(1+t_{1}+|\mathbf{t}|^{2}\right) e^{23} \\
& \rho(\mathbf{t}, r) \Omega_{5}^{1}=-t_{2}\left(e^{16}+|\mathbf{t}| e^{35}\right)+t_{1}\left(e^{26}+|\mathbf{t}| e^{45}\right) \\
& \rho(\mathbf{t}, r) \Omega_{6}^{1}=-t_{1}\left(e^{16}+|\mathbf{t}| e^{35}\right)-t_{2}\left(e^{26}+|\mathbf{t}| e^{45}\right) \\
& \rho(\mathbf{t}, r) \Omega_{4}^{3}=3|\mathbf{t}|^{2} e^{12}+t_{1}|\mathbf{t}|\left(e^{13}-e^{24}\right)+t_{2}|\mathbf{t}|\left(e^{14}+e^{23}\right)-|\mathbf{t}|^{2} e^{34} \\
& \rho(\mathbf{t}, r) \Omega_{5}^{3}=-t_{2}\left(|\mathbf{t}| e^{15}-e^{36}\right)+t_{1}\left(|\mathbf{t}| e^{25}-e^{46}\right) \\
& \rho(\mathbf{t}, r) \Omega_{6}^{3}=-t_{1}\left(|\mathbf{t}| e^{15}-e^{36}\right)-t_{2}\left(|\mathbf{t}| e^{25}-e^{46}\right),
\end{aligned}
$$


where $\rho(\mathbf{t}, r)=\frac{|\mathbf{t}|^{2}\left(1-|\mathbf{t}|^{2}\right)^{2}}{r^{2}}$. The other curvature 2-forms are given by the relations: $\Omega_{3}^{2}=-\Omega_{4}^{1}, \Omega_{4}^{2}=\Omega_{3}^{1}, \Omega_{5}^{2}=-\Omega_{6}^{1}, \Omega_{6}^{2}=\Omega_{5}^{1}, \Omega_{5}^{4}=-\Omega_{6}^{3}, \Omega_{6}^{4}=\Omega_{5}^{3}$, and $\Omega_{6}^{5}=$ $-\Omega_{2}^{1}-\Omega_{4}^{3}$.

Now, we have

$$
\begin{aligned}
\operatorname{tr} \Omega_{\mathbf{t}, r} \wedge \Omega_{\mathbf{t}, r} & =\sum_{1 \leq i<j \leq 6} \Omega_{j}^{i} \wedge \Omega_{j}^{i} \\
& =-4 r^{4} \frac{1+|\mathbf{t}|^{2}+2|\mathbf{t}|^{4}}{|\mathbf{t}|^{4}\left(1-|\mathbf{t}|^{2}\right)^{4}} e^{1234}=r^{4} \frac{1+|\mathbf{t}|^{2}+2|\mathbf{t}|^{4}}{|\mathbf{t}|^{2}\left(1-|\mathbf{t}|^{2}\right)^{4}} \eta_{\mathbf{t}}^{1 \overline{1} 2 \overline{2}}
\end{aligned}
$$

Taking the trivial instanton $A=0$ (see Remark 5.4 for solutions with non-flat instanton), we conclude that for each $\mathbf{t}$ such that $0<|\mathbf{t}|<1$, there exists a solution to the anomaly cancellation equation with positive $\alpha^{\prime}$. Indeed, from the equality

$$
\begin{aligned}
\frac{r^{2}\left(1+3|\mathbf{t}|^{2}\right)}{2\left(1-|\mathbf{t}|^{2}\right)^{2}} \eta_{\mathbf{t}}^{1 \overline{1} 2 \overline{2}} & =d T_{\mathbf{t}, r} \\
& =\frac{\alpha^{\prime}}{4}\left(\operatorname{tr} \Omega_{\mathbf{t}, r} \wedge \Omega_{\mathbf{t}, r}-\operatorname{tr} \Omega^{A} \wedge \Omega^{A}\right) \\
& =\alpha^{\prime} \frac{r^{4}\left(1+|\mathbf{t}|^{2}+2|\mathbf{t}|^{4}\right)}{4|\mathbf{t}|^{2}\left(1-|\mathbf{t}|^{2}\right)^{4}} \eta_{\mathbf{t}}^{1 \overline{1} 2 \overline{2}},
\end{aligned}
$$

we are led to the following positive value for the slope parameter:

$$
\alpha^{\prime}=\frac{2|\mathbf{t}|^{2}\left(1+3|\mathbf{t}|^{2}\right)\left(1-|\mathbf{t}|^{2}\right)^{2}}{r^{2}\left(1+|\mathbf{t}|^{2}+2|\mathbf{t}|^{4}\right)}>0 .
$$

\section{The Negative Slope Parameter Case}

In this section, we focus on the Strominger property for negative slope parameter $\alpha^{\prime}$.

The first solutions of this kind were obtained in [12] on the Iwasawa manifold by using a certain abelian instanton $A$ (see the proof of Theorem 5.1). In [20], it is proved that there is no invariant solution to the Strominger system with $\alpha^{\prime}>0$ with respect to the Chern, Strominger-Bismut, Levi-Civita or the $\nabla^{-}=\nabla^{g}-\frac{1}{2} H$ connections in the anomaly cancellation equation. However, to our knowledge, it is an open question if the Iwasawa manifold can admit or not any solution to the Strominger system with $\alpha^{\prime}>0$.

Explicit solutions with $\alpha^{\prime}<0$ on nilmanifolds, solvmanifolds, and on compact quotient of $\operatorname{SL}(2, \mathbb{C})$ are constructed in $[18,20,21,33,42]$, both with trivial or non-flat instanton, and with respect to a family of connections in the anomaly cancellation equation. On the other hand, in [23], Fu and Yau obtained solutions to the Strominger system with negative $\alpha^{\prime}$ on a class of complex 3-dimensional manifolds constructed by Goldstein and Prokushkin [27]. More recently, Phong, Picard, and Zhang solved in 
[34] the Fu-Yau equation with negative slope parameter in arbitrary dimensions and studied its relation to a certain modification of the Strominger system.

An interesting question is what are the differences and similarities of the geometric properties of the positive and the negative slope parameter cases. Notice that, a nonstability result similar to Theorem 3.2 in the case of negative $\alpha^{\prime}$ follows directly from [1] and [12]. Indeed, by [12], the Iwasawa manifold has a solution to the Strominger system with $\alpha^{\prime}<0$, and by [1] there are small holomorphic deformations of the Iwasawa manifold not admitting any balanced metric. However, we do not know of any result about closedness of the Strominger property for negative $\alpha^{\prime}$. Next we prove a similar result to Theorem 4.1 .

Theorem 5.1 The Strominger property with negative slope parameter is neither open nor closed under holomorphic deformations.

Proof As we noticed above, there are small deformations of the Iwasawa manifold showing that the Strominger property with $\alpha^{\prime}<0$ is not stable. In the Remark 5.3 below, we further study the compact complex manifolds $X^{s}$ constructed in Proposition 3.1, and show that any $X^{s}$ also admits a solution to the Strominger system with $\alpha^{\prime}<0$. Hence, one has a family of explicit examples of compact complex manifolds on which the property is not stable.

For the proof of non-closedness of the Strominger property with negative slope parameter, we will consider the family $X_{\mathbf{t}}$ constructed in the proof of Theorem 4.1. Next, we show that $X_{\mathbf{t}}$ admits a solution to the Strominger system with $\alpha^{\prime}<0$ for every $0<|\mathbf{t}|<1$.

Since the compact complex manifold $X_{\mathbf{t}}$ has the structure of a torus bundle over a complex 2-torus, we can consider the instanton constructed by Cardoso et al. [12]. They considered an abelian field strength configuration with (1,1)-form

$$
\begin{aligned}
\mathcal{F}= & \text { if } d z_{1} \wedge d \bar{z}_{1}-i f d z_{2} \wedge d \bar{z}_{2}+\mathrm{e}^{i \gamma} \sqrt{\frac{1}{4}-f^{2}} d z_{1} \wedge d \bar{z}_{2} \\
& -\mathrm{e}^{-i \gamma} \sqrt{\frac{1}{4}-f^{2}} d z_{2} \wedge d \bar{z}_{1},
\end{aligned}
$$

where the function $f$ satisfies

$$
i \partial_{z_{2}} f+\partial_{z_{1}}\left(\mathrm{e}^{-i \gamma} \sqrt{\frac{1}{4}-f^{2}}\right)=0, \quad i \partial_{z_{1}} f+\partial_{z_{2}}\left(\mathrm{e}^{i \gamma} \sqrt{\frac{1}{4}-f^{2}}\right)=0
$$

Under these conditions, one gets

$$
\operatorname{tr} \Omega^{A} \wedge \Omega^{A}=\mathcal{F} \wedge \mathcal{F}=-\frac{1}{2} d z_{1} \wedge d z_{2} \wedge d \bar{z}_{1} \wedge d \bar{z}_{2}
$$

Here $d z_{1}$ and $d z_{2}$ denote the (1,0)-forms at the level of the Lie group, which descend to the forms $\eta_{\mathbf{t}}^{1}$ and $|\mathbf{t}| \eta_{\mathbf{t}}^{2}$ on the compact nilmanifold $X_{\mathbf{t}}=\left(N, J_{\mathbf{t}}\right)$. Notice that the Hermitian-Yang-Mills equation is satisfied, i.e., 


$$
\Omega^{A} \wedge F_{\mathbf{t}, r}^{2}=0, \quad\left(\Omega^{A}\right)^{0,2}=\left(\Omega^{A}\right)^{2,0}=0 .
$$

Thus, by (5.1) we have $\operatorname{tr} \Omega^{A} \wedge \Omega^{A}=-\frac{1}{2} d z_{1} \wedge d z_{2} \wedge d \bar{z}_{1} \wedge d \bar{z}_{2}=\frac{|\mathbf{t}|^{2}}{2} \eta_{\mathbf{t}}^{1 \overline{1} 2 \overline{2}}$, and the anomaly cancellation equation becomes

$$
\begin{aligned}
\frac{r^{2}\left(1+3|\mathbf{t}|^{2}\right)}{2\left(1-|\mathbf{t}|^{2}\right)^{2}} \eta_{\mathbf{t}}^{1 \overline{1} 2 \overline{2}} & =d T_{\mathbf{t}, r} \\
& =\frac{\alpha^{\prime}}{4}\left(\operatorname{tr} \Omega_{\mathbf{t}, r} \wedge \Omega_{\mathbf{t}, r}-\operatorname{tr} \Omega^{A} \wedge \Omega^{A}\right) \\
& =\frac{\alpha^{\prime}}{4}\left(\frac{r^{4}\left(1+|\mathbf{t}|^{2}+2|\mathbf{t}|^{4}\right)}{|\mathbf{t}|^{2}\left(1-|\mathbf{t}|^{2}\right)^{4}}-\frac{|\mathbf{t}|^{2}}{2}\right) \eta_{\mathbf{t}}^{1 \overline{1} 2 \overline{2}}
\end{aligned}
$$

This implies that the slope parameter is given by

$$
\alpha^{\prime}=\frac{4 r^{2}|\mathbf{t}|^{2}\left(1+3|\mathbf{t}|^{2}\right)\left(1-|\mathbf{t}|^{2}\right)^{2}}{2 r^{4}\left(1+|\mathbf{t}|^{2}+2|\mathbf{t}|^{4}\right)-|\mathbf{t}|^{4}\left(1-|\mathbf{t}|^{2}\right)^{4}} .
$$

Therefore, on $X_{\mathbf{t}}, 0<|\mathbf{t}|<1$, we can take a balanced metric $F_{\mathbf{t}, r}$ in (4.6) with $r \in \mathbb{R}^{*}$ small enough such that $r^{4}<\frac{|\mathbf{t}|^{4}\left(1-|\mathbf{t}|^{2}\right)^{4}}{2\left(1+|\mathbf{t}|^{2}+2|\mathbf{t}|^{4}\right)}$, which ensures that $\alpha^{\prime}<0$. In conclusion, we have constructed a holomorphic family of compact complex manifolds $\left(X_{\mathbf{t}}\right)_{\mathbf{t} \in \Delta}$, where $\Delta=\{\mathbf{t} \in \mathbb{C}|| \mathbf{t} \mid<1\}$, such that $X_{\mathbf{t}}$ has holomorphically trivial canonical bundle for any $\mathbf{t} \in \Delta$, and

(i) $X_{\mathbf{t}}$ admits a solution to the Strominger system with $\alpha^{\prime}<0$ for every $\mathbf{t} \in \Delta-\{0\}$, but

(ii) $X_{0}$ does not admit any balanced Hermitian metric.

In particular, the Strominger property with negative slope parameter is not closed under holomorphic deformations.

Remark 5.2 In the proof of Proposition 3.1, we have considered the trivial instanton $A$ in the anomaly cancellation equation. It is worth to remark that one can construct solutions with non-flat instanton on every compact complex manifold $X^{s}=\left(M, J^{S}\right)$. For that, we must first enlarge the space of balanced $J^{s}$-Hermitian metrics (3.2) by

$$
F_{s, r}=\frac{i}{2}\left(\omega^{1 \overline{1}}+s^{2} \omega^{2 \overline{2}}+r^{2} \omega^{3 \overline{3}}\right)
$$

where $r \in \mathbb{R}^{*}$. In fact, $F_{s, r}^{2}$ is a closed form, so $F_{s, r}$ defines a 1-parameter family of balanced $J^{s}$-Hermitian metrics on $X^{s}$. A similar calculation as in the proof of Proposition 3.1 shows that the torsion $T_{s, r}=J^{s} d F_{s, r}$ and the curvature $\Omega_{s, r}$ of the Strominger-Bismut connection satisfy

$$
d T_{s, r}=r^{2} \frac{2 s^{2}+1}{s^{4}} \omega^{1 \overline{1} 2 \overline{2}}, \quad \operatorname{tr} \Omega_{s, r} \wedge \Omega_{s, r}=4 r^{4} \frac{4 s^{2}+1}{s^{4}} \omega^{1 \overline{1} 2 \overline{2}}
$$


Since the compact complex manifold $X^{s}$ has the structure of a torus bundle over a complex 2-torus, we can consider the instanton constructed in [12] and used in the proof of Theorem 5.1. In this case, we consider $d z_{1}$ and $d z_{2}$ the (1,0)-forms at the level of the Lie group, which descend to the forms $\omega^{1}$ and $s \omega^{2}$ on the compact nilmanifold $X^{s}=\left(M, J^{s}\right)$. Thus, by (5.1), we have tr $\Omega^{A} \wedge \Omega^{A}=-\frac{1}{2} d z_{1} \wedge d z_{2} \wedge d \bar{z}_{1} \wedge d \bar{z}_{2}=$ $\frac{s^{2}}{2} \omega^{1 \overline{1} 2 \overline{2}}$, and the anomaly cancellation equation becomes

$$
\begin{aligned}
r^{2} \frac{2 s^{2}+1}{s^{4}} \omega^{1 \overline{1} 2 \overline{2}} & =d T_{s, r} \\
& =\frac{\alpha^{\prime}}{4}\left(\operatorname{tr} \Omega_{s, r} \wedge \Omega_{s, r}-\operatorname{tr} \Omega^{A} \wedge \Omega^{A}\right) \\
& =\frac{\alpha^{\prime}}{4}\left(4 r^{4} \frac{4 s^{2}+1}{s^{4}}-\frac{s^{2}}{2}\right) \omega^{1 \overline{1} 2 \overline{2}} .
\end{aligned}
$$

This implies that $\alpha^{\prime}=\frac{8 r^{2}\left(2 s^{2}+1\right)}{8 r^{4}\left(4 s^{2}+1\right)-s^{6}}$. Therefore, on the compact complex manifold $X^{s}$ we can take a balanced metric $F_{s, r}$ in (5.3) with $r \in \mathbb{R}$ large enough such that $r^{4}>\frac{s^{6}}{8\left(4 s^{2}+1\right)}$, which ensures that $\alpha^{\prime}>0$.

Remark 5.3 Notice that, in Remark 5.2, taking $r$ small enough such that $0<r^{4}<$ $\frac{s^{6}}{8\left(4 s^{2}+1\right)}$, one has on every compact complex manifold $X^{s}$ a solution to the Strominger system with $\alpha^{\prime}<0$ in the anomaly cancellation equation. Thus, using the holomorphic deformation of $X^{s}$ constructed in Theorem 3.2, we get a family of examples for which the Strominger property with $\alpha^{\prime}<0$ is non-stable.

Remark 5.4 In the proof of Theorem 4.1, we have considered on $X_{\mathbf{t}}, 0<|\mathbf{t}|<1$, the trivial instanton $A$ in the anomaly cancellation equation. It is worth to remark that one can construct solutions with $\alpha^{\prime}>0$ and non-flat instanton. Indeed, following the proof of Theorem 5.1, it is enough to take a balanced metric $F_{\mathbf{t}, r}$ in (4.6) with $r \in \mathbb{R}^{*}$ large enough such that $r^{4}>\frac{\left.|\mathbf{t}|^{4}(1-\mid \mathbf{t})^{2}\right)^{4}}{2\left(1+|\mathbf{t}|^{2}+2|\mathbf{t}|^{4}\right)}$, which ensures $\alpha^{\prime}>0$.

Acknowledgements This work has been partially supported by the projects MINECO (Spain) MTM201458616-P, Gobierno de Aragón/Fondo Social Europeo-Grupo Consolidado E15 Geometría, and by Fundación Bancaria Ibercaja-Fundación CAI-Universidad de Zaragoza, Programa de Estancias de Investigación, Contracts DH 12/3/12.12.2017 and Contract 80-10-33/2017 with the Sofia University "St.Kl.Ohridski." S.I. thanks the University of Zaragoza for the support during his visit to the Department of Mathematics, and L.U. thanks the University of Sofia "St. K1. Ohridski" for the hospitality and financial support provided while visiting the Faculty of Mathematics and Informatics. The authors wish to thank the referee for useful comments and suggestions.

\section{References}

1. Alessandrini, L., Bassanelli, G.: Small deformations of a class of compact non-Kähler manifolds. Proc. Am. Math. Soc. 109, 1059-1062 (1990)

2. Anderson, L.B., Gray, J., Sharpe, E.: Algebroids, heterotic moduli spaces and the Strominger system. J. High Energy Phys. 07, 037 (2014) 
3. Andreas, B., García-Fernández, M.: Solutions of the Strominger system via stable bundles on CalabiYau threefolds. Commun. Math. Phys. 315, 153-168 (2012)

4. Andreas, B., García-Fernández, M.: Note on solutions of the Strominger system from unitary representations of cocompact lattices of SL(2, C). Commun. Math. Phys. 332, 1381-1383 (2014)

5. Angella, D., Ugarte, L.: On small deformations of balanced manifolds. Differ. Geom. Appl. 54, 464474 (2017)

6. Angella, D., Franzini, M.G., Rossi, F.A.: Degree of non-Kählerianity for 6-dimensional nilmanifolds. Manuscr. Math. 148(1-2), 177-211 (2015)

7. Becker, K., Tseng, L.-S.: Heterotic flux compactifications and their moduli. Nucl. Phys. B 741, 162-179 (2006)

8. Becker, K., Becker, M., Dasgupta, K., Green, P.S., Sharpe, E.: Compactifications of heterotic strings on non-Kähler complex manifolds: II. Nucl. Phys. B 678, 19-100 (2004)

9. Bergshoeff, E.A., de Roo, M.: The quartic effective action of the heterotic string and supersymmetry. Nucl. Phys. B 328, 439 (1989)

10. Bismut, J.-M.: A local index theorem for non-Kähler manifolds. Math. Ann. 284, 681-699 (1989)

11. Bogomolov, F.A.: Hamiltonian Kähler manifolds. Dolk. Akad. Nauk SSSR 243, 1101-1104 (1978)

12. Cardoso, G.L., Curio, G., Dall'Agata, G., Lust, D., Manousselis, P., Zoupanos, G.: Non-Käehler string back-grounds and their five torsion classes. Nucl. Phys. B 652, 5-34 (2003)

13. Ceballos, M., Otal, A., Ugarte, L., Villacampa, R.: Invariant complex structures on 6-nilmanifolds: classification, Frölicher spectral sequence and special Hermitian metrics. J. Geom. Anal. 26, 252-286 (2016)

14. de la Ossa, X.C., Svanes, E.E.: Holomorphic bundles and the moduli space of $N=1$ supersymmetric heterotic compactifications. J. High Energy Phys. 10, 123 (2014)

15. de la Ossa, X.C., Svanes, E.E.: Connections, field redefinitions and heterotic supergravity. J. High Energy Phys. 12, 008 (2014)

16. de la Ossa, X.C., Hardy, E., Svanes, E.E.: The heterotic superpotential and moduli. J. High Energy Phys. 01, 049 (2016)

17. Fei, T.: A construction of non-Kähler Calabi-Yau manifolds and new solutions to the Strominger system. Adv. Math. 302, 529-550 (2016)

18. Fei, T., Yau, S.-T.: Invariant solutions to the Strominger system on complex Lie groups and their quotients. Commun. Math. Phys. 338, 1183-1195 (2015)

19. Fei, T., Huang, Z., Picard, S.: A construction of infinitely many solutions to the Strominger system. arXiv:1703.10067 [math.DG]

20. Fernández, M., Ivanov, S., Ugarte, L., Villacampa, R.: Non-Kähler heterotic string compactifications with non-zero fluxes and constant dilaton. Commun. Math. Phys. 288, 677-697 (2009)

21. Fernández, M., Ivanov, S., Ugarte, L., Vassilev, D.: Non-Kaehler heterotic string solutions with nonzero fluxes and non-constant dilaton. J. High Energy Phys. 06, 073 (2014)

22. Fino, A., Grantcharov, G.: Properties of manifolds with skew-symmetric torsion and special holonomy. Adv. Math. 189, 439-450 (2004)

23. Fu, J.-X., Yau, S.-T.: A Monge-Ampère type equation motivated by string theory. Commun. Anal. Geom. 15, 29-76 (2007)

24. Fu, J.-X., Yau, S.-T.: The theory of superstring with flux on non-Kähler manifolds and the complex Monge-Ampère equation. J. Differ. Geom. 78, 369-428 (2008)

25. García-Fernández, M.: Lectures on the Strominger system. arXiv:1609.02615 [math.DG]

26. García-Fernández, M., Rubio, R., Tipler, C.: Infinitesimal moduli for the Strominger system and generalized Killing spinors. Math. Ann. 369, 539-595 (2017)

27. Goldstein, E., Prokushkin, S.: Geometric model for complex non-Käehler manifolds with SU(3) structure. Commun. Math. Phys. 251, 65-78 (2004)

28. Hull, C.M.: Compactifications of the heterotic superstring. Phys. Lett. B 178(4), 357-364 (1986)

29. Latorre, A., Ugarte, L., Villacampa, R.: On the Bott-Chern cohomology and balanced Hermitian nilmanifolds. Int. J. Math. 25(6), 1450057 (2014). 24 pp

30. Li, J., Yau, S.-T.: The existence of supersymmetric string theory with torsion. J. Differ. Geom. 70(1), 143-181 (2005)

31. Maclaughlin, C., Pedersen, H., Poon, Y.S., Salamon, S.: Deformation of 2-step nilmanifolds with abelian complex structures. J. Lond. Math. Soc. 73, 173-193 (2006)

32. Michelsohn, M.L.: On the existence of special metrics in complex geometry. Acta Math. 149(3-4), 261-295 (1982) 
33. Otal, A., Ugarte, L., Villacampa, R.: Invariant solutions to the Strominger system and the heterotic equations of motion. Nucl. Phys. B 920, 442-474 (2017)

34. Phong, D.H., Picard, S., Zhang, X.: The Fu-Yau equation with negative slope parameter. Invent. Math. 209, 541-576 (2017)

35. Phong, D.H., Picard, S., Zhang, X.: The anomaly flow and the Fu-Yau equation. arXiv:1610.02740 [math.DG]

36. Popovici, D.: Deformation openness and closedness of various classes of compact complex manifolds; examples. Ann. Sc. Norm. Super. Pisa Cl. Sci. (5) 13(2), 255-305 (2014)

37. Rollenske, S.: Lie-algebra Dolbeault-cohomology and small deformations of nilmanifolds. J. Lond. Math. Soc. 79, 346-362 (2009)

38. Salamon, S.: Complex structures on nilpotent Lie algebras. J. Pure Appl. Algebra 157, 311-333 (2001)

39. Strominger, A.: Superstrings with torsion. Nucl. Phys. B 274, 253 (1986)

40. Tian, G.: Smoothness of the Universal Deformation Space of Compact Calabi-Yau Manifolds and Its Petersson-Weil Metric, Mathematical Aspects of String Theory (San Diego, CA, 1986). Advanced Series in Mathematical Physics, pp. 629-646. World Scientific Publishing, Singapore (1987)

41. Todorov, A.N.: The Weil-Petersson geometry of the moduli space of SU( $n=3)$ (Calabi-Yau) manifolds. I. Commun. Math. Phys. 126, 325-346 (1989)

42. Ugarte, L., Villacampa, R.: Balanced Hermitian geometry on 6-dimensional nilmanifolds. Forum Math. 27, 1025-1070 (2015)

43. Yau, S.-T.: On the Ricci curvature of a compact Kähler manifold and the complex Monge-Ampère equation, I. Commun. Pure Appl. Math. 31, 339-411 (1978) 